\title{
A Central Mass in a Stationary Vacuum without Spherical or Axial Symmetry
}

\author{
William Davidson \\ Mathematics and Statistics Department, University of Otago, Dunedin, New Zealand \\ Correspondence should be addressed to William Davidson; william.davidson5@virginmedia.com
}

Received 15 March 2013; Accepted 22 May 2013

Academic Editors: H. Dehnen and D. Kieda

Copyright (c) 2013 William Davidson. This is an open access article distributed under the Creative Commons Attribution License, which permits unrestricted use, distribution, and reproduction in any medium, provided the original work is properly cited.

\begin{abstract}
A vacuum spacetime with a central mass is derived as a stationary solution to Einstein's equations. The vacuum metric has a geodesic, shear-free, expanding, and twisting null congruence $k$ and thus is algebraically special. The properties of the metric are calculated. In particular, it is shown that the spacetime has an event horizon inside which there is a black hole. The metric is neither spherically nor axially symmetric. It is therefore in interesting contrast with the majority of metrics featuring a central mass which have one or more of these symmetry properties. The metric reduces to the Schwarzschild case when a certain parameter is set to zero.
\end{abstract}

\section{Introduction}

In this paper, we present a special solution of Einstein's equations which can be described as a stationary vacuum spacetime with a central mass singularity without spherical or axial symmetry. Apart from the mass $m$, the metric will depend on a single parameter $a$, and it reduces to the Schwarzschild solution when $a$ is set to zero.

The character of this spacetime will be such that the Weyl tensor has a multiple null eigenvector $k$ forming a geodesic, shear-free, and diverging twisting congruence. Thus, in terms of the Newman-Penrose spin coefficients, we can write as properties of $k$ the following:

$$
\kappa=\sigma=0, \quad \rho=-(\Theta+i \omega) \neq 0,
$$

$\Theta$ being the divergence and $\omega$ the twist. Since the solution to be derived is vacuum, the previous properties mean that the metric will be algebraically special, so that of the Weyl complex coefficients we have

$$
\psi_{0}=\psi_{1}=0 .
$$

Reference to the spacetime will be via a complex null tetrad $m, m b a r, n$, and $k$ with labels $1,2,3$, and 4, respectively, $k$ being the vector described earlier. The field equations for a vacuum metric that admits a geodesic, shear-free, and diverging null congruence $k$ were obtained by Kerr [1] and I. Robinson and J. R. Robinson [2] and further developed by Debney et al. [3]. A different approach to obtaining vacuum solutions was made by Kinnersley [4]. Here we follow mainly the methods of [5] and finally derive our solution in the specific form of (16).

Since the central mass will be without spherical or axial symmetry, in contrast with the well-known GR vacuum solutions with mass singularity, it is believed that the derived spacetime in its final form (16) is of significant interest.

\section{Basic Equations}

In relation to the null tetrad, we will employ coordinates $x^{i}=x, y, r$, and $u$ for $i=1,2,3$, and 4 , respectively. The spacelike coordinate $x$ is complex, $y$ being its conjugate, $r$ an affine parameter along the $k$ lines, and $u$ a retarded time. We will use the notation of [5, chapters 29 and 30]. The metric signature will be taken to be +2 and the speed of light and the Einstein gravitational constant to be unity. The spacetime metric, in terms of 1 -forms relating the null tetrad to the $x^{i}$ system, is $[2,3,5]$

$$
d s^{2}=2 \omega^{1} \omega^{2}-2 \omega^{3} \omega^{4},
$$


where

$$
\begin{gathered}
\omega^{1}=-\frac{d x}{(P \bar{\rho})}, \quad \omega^{2}=-\frac{d y}{(P \rho)}, \\
\omega^{3}=d u+L d x+\bar{L} d y, \\
\omega^{4}=d r+W d x+\bar{W} d y+H \omega^{3} .
\end{gathered}
$$

In the $x^{i}$ system, we then have

$$
\begin{gathered}
m^{i}=(-P \bar{\rho}, 0, P W \bar{\rho}, P L \bar{\rho}), \\
\bar{m}^{i}=(0,-P \rho, P \bar{W} \rho, P \bar{L} \rho), \\
n^{i}=(0,0,-H, 1), \\
k^{i}=(0,0,1,0) .
\end{gathered}
$$

Here, for the case of a stationary solution,

$$
\begin{gathered}
\rho=-\frac{1}{(r+i \Sigma)}, \\
\Sigma=-\frac{i P^{2}(\bar{\partial} L-\partial \bar{L})}{2}, \\
W=i \partial \Sigma, \\
\partial=\partial_{x} .
\end{gathered}
$$

The coefficient of $d u^{2}$ is $-2 H$, where

$$
\begin{gathered}
H=\frac{K}{2}-\frac{(m r+M \Sigma)}{\left(r^{2}+\Sigma^{2}\right)}, \\
K=2 P^{2} \operatorname{Re}(\partial \bar{\partial} \ln P), \\
M=\Sigma K+P^{2} \operatorname{Re}(\partial \bar{\partial} \Sigma) .
\end{gathered}
$$

Here $P, K$, and $M$ are real functions and $L$ a complex function, of $x$ and $y . H$ is a real function and $W$ a complex function, of $x, y$, and $r . m$ is a constant $(>0)$. A subscripted comma indicates partial differentiation. For a stationary vacuum spacetime, the equations are

$$
\begin{gathered}
M=\text { constant }, \\
\left(\partial+2 P^{-1} \partial P\right) \partial C=0,
\end{gathered}
$$

where

$$
C=\bar{\partial}\left(P^{-1} \bar{\partial} P\right)+\left(P^{1} \bar{\partial} P\right)^{2} .
$$

Calculations will be made in the tetrad frame whose metric coefficients are

$$
g_{a b}=\left[\begin{array}{cccc}
0 & 1 & 0 & 0 \\
1 & 0 & 0 & 0 \\
0 & 0 & 0 & -1 \\
0 & 0 & -1 & 0
\end{array}\right]
$$

\section{A Stationary Vacuum Spacetime}

We take $L$ and $P$ to be

$$
\begin{gathered}
P=\frac{x y}{2}+1, \\
L=-\frac{i a}{P^{2}} \quad(a=\text { real const }>0) .
\end{gathered}
$$

Referring to (7), we find that

$$
M=0, \quad K=1,
$$

$$
H=\frac{a^{2}(x+y)^{2}+\left(r^{2}-2 m r\right)(x y+2)^{2}}{2\left[a^{2}(x+y)^{2}+r^{2}(x y+2)^{2}\right]} \text {. }
$$

Calculation of the Riemann tensor showed that all components vanish when $r \rightarrow \infty$. The metric is therefore flat at infinity. It was verified that $\psi_{0}=\psi_{1}=0$, and in fact calculation leads to a Petrov type $\mathrm{D}$. The vacuum equations (8) are evidently satisfied, and indeed all components of the Ricci tensor vanish confirming a vacuum metric.

For the curvature of the $x, y 2$-surface, a function depending on $x$ and $y$ as well as $r$ was obtained. It follows that the metric is neither spherically symmetric nor axisymmetric (see also Section 4).

From the Newman-Penrose spin coefficient $\rho$, we obtain for the divergence and twist of the $k$ lines

$$
\begin{aligned}
& \Theta=\frac{r(x y+2)^{2}}{r^{2}(x y+2)^{2}+a^{2}(x+y)^{2}}, \\
& \omega=\frac{-a(x+y)(x y+2)}{r^{2}(x y+2)^{2}+a^{2}(x+y)^{2}},
\end{aligned}
$$

respectively.

\section{The Metric in Real Coordinates}

If we make the transformation

$$
\begin{aligned}
& x=\sqrt{2} \tan \left(\frac{\theta}{2}\right) e^{i \xi}, \\
& y=\sqrt{2} \tan \left(\frac{\theta}{2}\right) e^{-i \xi},
\end{aligned}
$$


then the metric coefficients can be expressed in terms of real coordinates $\theta, \xi$, and $r$, numbered 1,2 , and 3 , respectively. Thus, the transformed metric coefficients $g_{i j}$ become

$$
\begin{aligned}
& g_{\theta \theta}=\left(4 r^{4}+2 a^{2} r(1+\cos \theta)\right. \\
& \times\left\{r(1-\cos \theta)\left(1+\cos ^{2} \xi\right)+2 m(1+\cos \theta) \sin ^{2} \xi\right\} \\
& \left.+a^{4} \sin ^{4} \theta \cos ^{2} \xi\right) \\
& \times\left(4 r^{2}+2 a^{2} \sin ^{2} \theta \cos ^{2} \xi\right)^{-1}, \\
& g_{\theta \xi}=\frac{2 a^{2} m r \sin \theta(1+\cos \theta)^{2} \sin \xi \cos \xi}{2 r^{2}+a^{2} \sin ^{2} \theta \cos ^{2} \xi}, \\
& g_{\xi \xi}=\left(2 r^{4} \sin ^{2} \theta+a^{2} r \sin ^{2} \theta(1+\cos \theta) \cos ^{2} \xi\right. \\
& \times\{r(1-\cos \theta)+2 m(1+\cos \theta)\}) \\
& \times\left(2 r^{2}+a^{2} \sin ^{2} \theta \cos ^{2} \xi\right)^{-1}, \\
& g_{\theta r}=-\frac{a(1+\cos \theta) \sin \xi}{\sqrt{2}}, \\
& g_{\xi r}=-\frac{a(1+\cos \theta) \sin \theta \cos \xi}{\sqrt{2}}, \\
& g_{\theta u}=\left(a \operatorname { s i n } \xi \left\{4 m r(1+\cos \theta)-2 r^{2} \cos \theta\right.\right. \\
& \left.\left.-a^{2} \sin ^{2} \theta \cos \theta \cos ^{2} \xi\right\}\right) \\
& \times\left(\sqrt{2}\left\{2 r^{2}+a^{2} \sin ^{2} \theta \cos ^{2} \xi\right\}\right)^{-1}, \\
& g_{\xi u}=-\frac{2 a \sin \theta \cos \xi\left\{r^{2}-2 m r(1+\cos \theta)\right\}+a^{3} \sin ^{3} \theta \cos ^{3} \xi}{\sqrt{2}\left(2 r^{2}+a^{2} \sin ^{2} \theta \cos ^{2} \xi\right)}, \\
& g_{r r}=0, \\
& g_{r u}=-1, \\
& g_{u u}=-\frac{2 r^{2}-4 m r+a^{2} \sin ^{2} \theta \cos ^{2} \xi}{2 r^{2}+a^{2} \sin ^{2} \theta \cos ^{2} \xi} \text {. }
\end{aligned}
$$

We note that if we set $a=0$, the metric reduces to the Schwarzschild spacetime (in Eddington-Finkelstein coordinates). In terms of these coordinates, the divergence and twist of the $k$ lines, given by (14), have the expressions

$$
\begin{gathered}
\Theta=\frac{2 r}{2 r^{2}+a^{2} \sin ^{2} \theta \cos ^{2} \xi}, \\
\omega=-\frac{\sqrt{2} a \sin \theta \cos \xi}{2 r^{2}+a^{2} \sin ^{2} \theta \cos ^{2} \xi} .
\end{gathered}
$$

Also, it may be verified that the component $g_{u u}$ is the transform of $-2 H$ where $H$ is given in (13).
The hypersurfaces $r=r_{+}$and $r=r_{-}$which satisfy the relation

$$
2 r^{2}-4 m r+a^{2}=0
$$

are null surfaces. They form horizons

$$
\begin{aligned}
& r_{+}=m\left(1+\sqrt{1-\frac{a^{2}}{2 m^{2}}}\right), \\
& r_{-}=m\left(1-\sqrt{1-\frac{a^{2}}{2 m^{2}}}\right),
\end{aligned}
$$

and they are real for

$$
0<a<\sqrt{2} m .
$$

In this case, $r=r_{+}$is an event horizon and $r$ will be spacelike in its exterior but timelike in the region $r_{-}<r<r_{+}$(where $u$ will be spacelike).

From the metric, we see that a radial light ray $(d s=d \theta=$ $d \xi=0)$ which satisfies

$$
-2 d r+\frac{\left(2 r^{2}-4 m r+a^{2} \sin ^{2} \theta \cos ^{2} \xi\right)}{2 r^{2}+a^{2} \sin ^{2} \theta \cos ^{2} \xi} d u=0
$$

has $d r / d u>0$ in the region $r>r_{+}$, where $2 r^{2}-4 m r+$ $a^{2} \sin ^{2} \theta \cos ^{2} \xi>0$. However, in the region $r_{-}<r<r_{+}$where the factor $2 r^{2}-4 m r+a^{2} \sin ^{2} \theta \cos ^{2} \xi<0$, we have $d u / d r<0$, so that the light ray, and indeed all timelike particle motions, can only be inwards and never outwards. Thus, the metric has a black hole if $0<a<\sqrt{2} m$. Setting $a=0$, we get the familiar results for the black hole in the Schwarzschild solution.

We may verify in these coordinates that the Riemannian curvature of the $\theta, \xi$ 2-space ( $r=$ const; $u=$ const) depends on $\theta$ and $\xi$ as well as $r$, so that the metric is not spherically symmetric or axially symmetric. For the curvature, we derive

$$
\begin{aligned}
K_{\theta \xi}=( & 2 m\left(-3 a^{2} \sin ^{2} \theta \cos ^{2} \xi+2 r^{2}\right) \\
\times & \left\{a^{2}\left[2 r^{2}\left(5+6 \cos \theta+\cos ^{2} \theta\right)-4 m r(1+\cos \theta)^{2}\right]\right. \\
& \left.\left.+3 a^{4}(1+\cos \theta)^{2}+8 r^{4}\right\}\right) \\
\times & \left(\left(2 r^{2}+a^{2} \sin ^{2} \theta \cos ^{2} \xi\right)^{3}\right. \\
\times & \left.\left\{a^{2}\left[r \sin ^{2} \theta+2 m(1+\cos \theta)^{2}\right]+2 r^{3}\right\}\right)^{-1} .
\end{aligned}
$$

When $a=0$, this result becomes $2 m / r^{3}$, as appropriate to the Schwarzschild spacetime. The only Killing vector, when $a \neq 0$, is $\partial_{u}$ reflecting the stationary character of the solution.

\section{Conclusion}

In this paper, we have derived and described the properties of a stationary vacuum metric which has no spatial symmetry 
but reduces to the Schwarzschild spacetime when the parameter $a$ is set to zero. The spacetime is algebraically special and possesses a null congruence $k$ which is geodesic, shear-free, and whose divergence and twist are calculated. Subject to the relation $0<a<\sqrt{2} m$, there is shown to be an event horizon inside which there is a black hole. These features are believed to be of critical interest since they are in contrast with most previous discussions which have concerned a central mass in a spherically or axially symmetric environment.

\section{References}

[1] R. P. Kerr, "Gravitational field of a spinning mass as an example of algebraically special metrics," Physical Review Letters, vol. 11, no. 5, pp. 237-238, 1963.

[2] I. Robinson and J. R. Robinson, "Vacuum metrics without symmetry," International Journal of Theoretical Physics, vol. 2, no. 3, pp. 231-242, 1969.

[3] G. C. Debney, R. P. Kerr, and A. Schild, "Solutions of the Einstein and Einstein-Maxwell equations," Journal of Mathematical Physics, vol. 10, no. 10, pp. 1842-1854, 1969.

[4] W. Kinnersley, "Type D vacuum metrics," Journal of Mathematical Physics, vol. 10, no. 7, pp. 1195-1203, 1969.

[5] H. Stephani, D. Kramer, M. A. H. MacCallum, C. Hoenselaers, and E. Herlt, Exact Solutions of Einstein's Field Equations, Cambridge University Press, Cambridge, UK, 2003. 

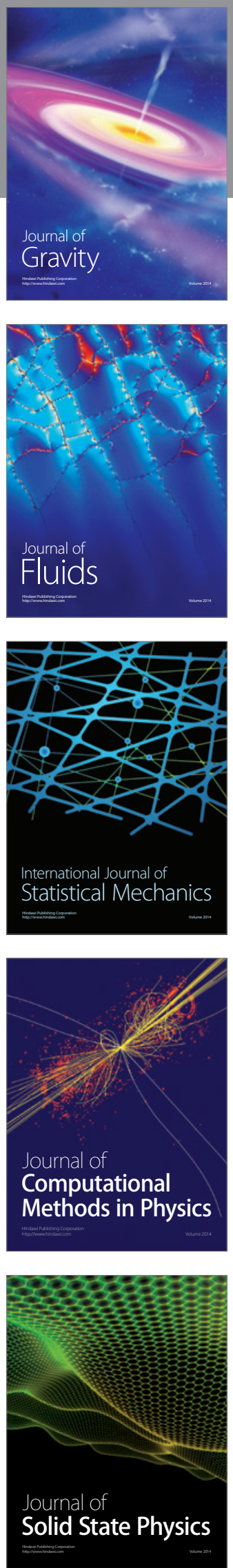

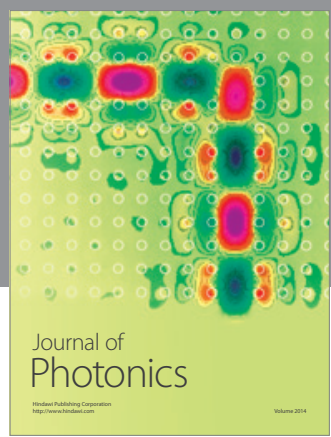

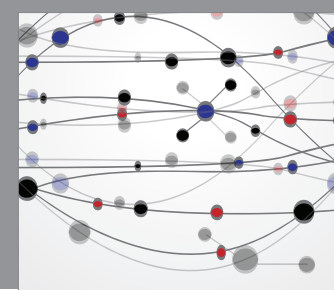

The Scientific World Journal

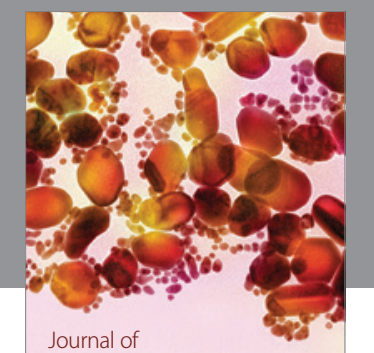

Soft Matter
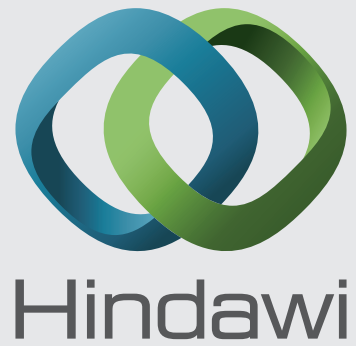

Submit your manuscripts at

http://www.hindawi.com
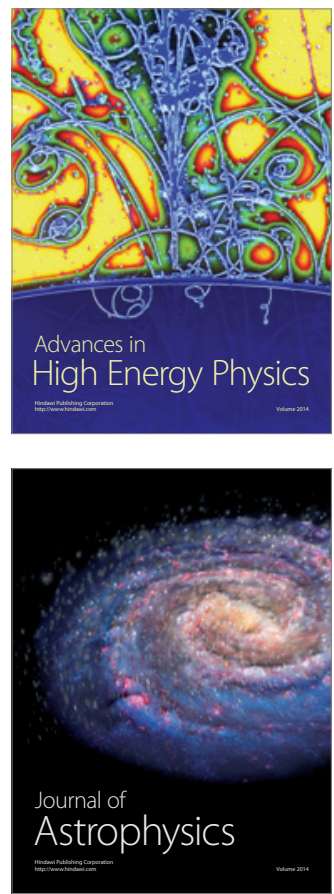
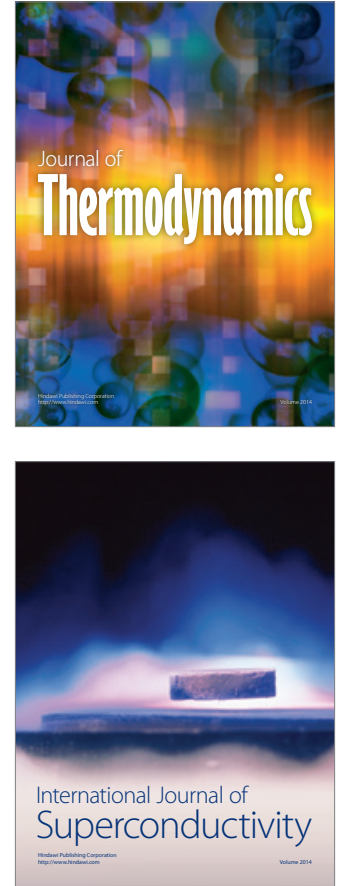
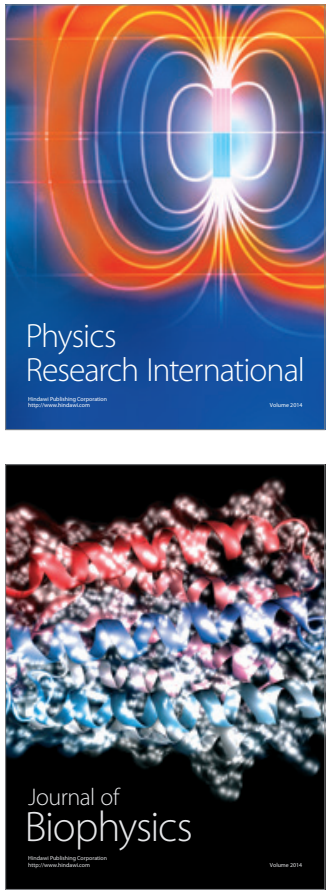
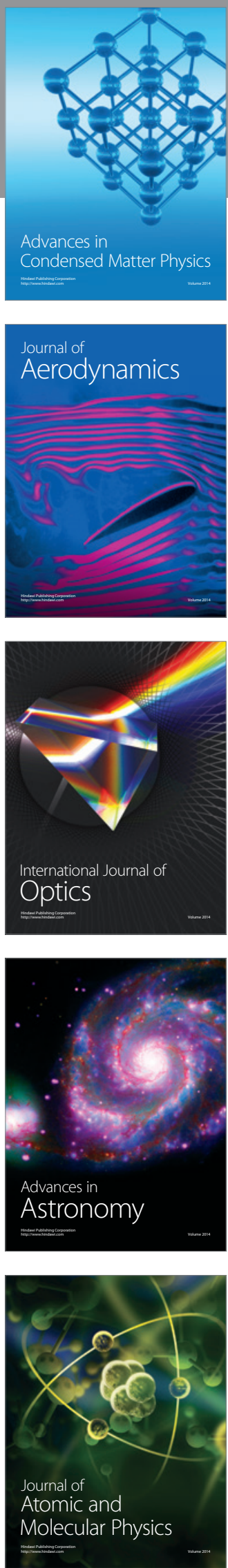